\author{
Sección Control / Control
}

Artículos de investigación / Research paper

\title{
Capacidad depredadora de Cathartus quadricollis y Ahasverus advena (Coleoptera: Silvanidae) sobre Hypothenemus hampei (Coleoptera: Curculionidae) en laboratorio
}

\author{
Predatory capacity of Cathartus quadricollis and Ahasverus advena (Coleoptera: Silvanidae) on Hypothenemus hampei
} (Coleoptera: Curculionidae) under laboratory conditions

\author{
LAURA A. LAITON J. ${ }^{1}$, LUIS MIGUEL CONSTANTINO ${ }^{2}$ y PABLO BENAVIDES ${ }^{3}$
}

\begin{abstract}
Resumen: La broca del café ocasiona daños económicos y deterioro en la calidad del grano. Se reproduce en los frutos que quedan después de las cosechas. En Colombia se han reportado depredadores nativos que atacan a esta plaga, entre ellos los coleópteros Ahasverus advena y Cathartus quadricollis. Esta investigación tuvo como objetivo evaluar la depredación de estas dos especies sobre la broca, en condiciones de laboratorio en Cenicafé, Manizales. En cuatro bioensayos independientes se liberaron los depredadores, tanto adultos como larvas, en proporciones de 1,3 y 5 por grano infestado. Se determinó la disminución de los estados de la broca al interior de los frutos después de establecidos los tratamientos. Las unidades experimentales estuvieron conformadas por 10 almendras de café infestadas con broca por 18 días, con tres orificios de entrada, en un recipiente de vidrio donde se aplicaron los cuatro tratamientos bajo un diseño completamente al azar con diez repeticiones. Se utilizó un testigo absoluto para cada bioensayo. Se encontró que tanto los adultos como las larvas de $A$. advena disminuyeron significativamente la broca entre $63,2 \%$ y $42,3 \%$ menos con respecto al testigo, siendo mayor la capacidad depredadora cuando se utilizaron tres individuos por grano brocado. Igualmente, C. quadricollis disminuyó de broca hasta 46,2 \% y $69 \%$ cuando se liberaron tres adultos y cinco larvas por grano, respectivamente.
\end{abstract}

Palabras clave: Control biológico, aumentación, depredación, calidad del café.

\begin{abstract}
The coffee berry borer (CBB) causes economic damage and quality deterioration of coffee. CBB reproduces inside the fruit that remain after harvesting. In Colombia, native predators have been reported attacking this pest, among them the coleopterans Ahasverus advena and Cathartus quadricollis. This study, which was carried out under lab conditions at Cenicafé, was evaluated the predation of these species on CBB. Adults and larvae of the two predator species were released in ratios of 1, 3 and 5 per infested coffee bean (the treatments) in four independent bioassays. The decrease in $\mathrm{CBB}$ populations inside the beans after establishing treatments (the response variables) was determined. The Experimental Units consisted of 10 infested coffee grains observed over 18 days with three infesting holes inside a glass container where the four treatments were applied under a completely randomized design with ten replicates. An absolute control without predators was used for each bioassay. A. advena adults and larvae significantly decreased CBB up to $63.2 \%$ and $42.3 \%$ with respect to the control, presenting a higher predation capacity when three predators per grain were released. In addition, C. quadricollis decreased CBB population up to $46.2 \%$ and $69 \%$ when three adults and five larvae per grain were released, respectively.
\end{abstract}

Key words: Biological control, augmentation, predation, coffee quality.

\section{Introducción}

La broca del café Hypothenemus hampei (Ferrari, 1867) (Coleoptera: Curculionidae) es el principal problema entomológico para la caficultura mundial, ya que se alimenta y reproduce en la almendra, ocasiona pérdida de su calidad y peso, causa la caída prematura de los frutos en formación y provoca considerables pérdidas económicas. Desde su llegada a Colombia en 1988, en ausencia de sus enemigos naturales, la broca del café se estableció rápidamente alcanzando niveles altos de población, particularmente en zonas bajas con temperaturas superiores a $21^{\circ} \mathrm{C}$ (Bustillo 2007; Benavides et al. 2013). El uso continuo e indiscriminado de insecticidas como única medida de control no es recomendable debido a los problemas ambientales que generan, al desarrollo de re- sistencia en la broca y su ineficacia una vez el insecto está en el interior del fruto (Bustillo 2007; Benavides et al. 2013). A partir de la llegada de la broca a Colombia, Cenicafé implementó una estrategia de manejo integrado de la broca (MIB), que involucra medidas de control cultural, biológico, legal y químico, para mantener las poblaciones por debajo del nivel de daño económico (5\%) en el cultivo (Bustillo et al. 1998).

En África Central, región de origen de la broca, existen varios enemigos naturales que regulan sus poblaciones, entre ellos los parasitoides Prorops nasuta (Waterston, 1923), Cephalonomia stephanoderis (Betrem, 1961) (Hymenoptera: Bethylidae), Phymastichus coffea (La Salle, 1990) (Hymenoptera: Eulophidae) y Heterospilus coffeicola (Schmiedknecht, 1923) (Hymenoptera: Braconidae), el depredador Karnyothrips flavipes (Jones, 1912) (Thysanoptera:

${ }^{1}$ I. A. Asistente de Investigación, Cenicafé, Manizales, Colombia, alexandra.laiton@cafedecolombia.com. ${ }^{2}$ Entomólogo, M. Sc. Investigador Científico II, Cenicafé, Manizales, Colombia, luismiguel.constantino@cafedecolombia.com. ${ }^{3}$ I. A. Ph. D. Investigador Científico III, Cenicafé, Manizales, Colombia, pablo.benavides@cafedecolombia.com. Autor por correspondencia: Pablo Benavides. Investigador Científico III, Cenicafé, Manizales, Colombia,pablo.benavides@cafedecolombia.com. 
Phaelothripidae) y el hongo entomopatógeno Beauveria bassiana (Balsamo) (Vuillemin, 1912) (Hypocreales: Clavicipitaceae) (Bustillo et al. 2002; Benavides 2008; Jaramillo 2008; Vega et al. 2009). En América hay reportes de los parasitoides Cephalonomia hyalinipennis (Ashmead, 1893) (Hymenoptera: Bethylidae) en México (PérezLachaud 1998) y Cryptoxilos sp. (Hymenoptera: Braconidae) en Colombia (Bustillo et al. 2002). Los insectos parasitoides $\mathrm{y}$ algunos depredadores encontrados dentro de los frutos brocados de café, tienen la habilidad de penetrar por el orificio de entrada que hace la broca en el fruto y buscar sus presas a través de las galerías y túneles. Entre los depredadores se tienen reportados en Colombia varios insectos que atacan los estados inmaduros y adultos en los frutos del árbol y del suelo. Las especies más importantes reportadas en la literatura pertenecen a las familias Formicidae (Hymenoptera), Anthocoridae (Hemiptera) y Silvanidae (Coleoptera). Dos especies nativas de Silvanidae, se han registrado dentro de las galerías y túneles en granos infestados de broca, siendo identificadas por Bustillo et al. (2002) y Vera et al. (2007) como Cathartus quadricollis Guerin-Meneville (Reiche, 1854) y Monanus sp. En condiciones de laboratorio, adultos colectados en campo de ambas especies, alcanzaron porcentajes de depredación de larvas y huevos de broca mayores al $60 \%$ (Vera et al. 2007). En 2013, Constantino reportó a Ahasverus advena (Waltl, 1832) y Europs sp., otras dos especies de Silvanidae como depredadores de estados biológicos de broca en Colombia (Cenicafé 2013).

Recientemente, Follet et al. (2016) evaluaron la capacidad depredadora de adultos de C. quadricollis con pruebas de depredación directa sobre estados biológicos de broca, donde se expusieron 20 estados de broca por cada tres depredadores adultos obteniendo 77,5\% de depredación en huevos, 55,0\% en larvas, $53,0 \%$ en pupas y $15,5 \%$ en adultos.

Tanto C. quadricollis como A. advena se encuentran de manera abundante en campo, además son fáciles de manipular en condiciones de laboratorio. Cathartus quadricollis es conocido vulgarmente como "cucarroncito cuellicuadrado" de los granos. Es especie de distribución pantropical y subtropical que se alimenta principalmente de granos de maíz partidos o dañados, granos de trigo, yuca seca y harina (Tróchez 1987). El adulto es de forma aplanada, oblongo, de color castaño rojizo, con una longitud de 2,5 $\mathrm{mm}$. El pronoto del cuello es de forma cuadrada con bordes lisos (Tróchez 1987). Ahasverus advena, se le conoce comúnmente como el cucarroncito aplanado menor de los granos. Se encuentra distribuido en todo el mundo en las zonas templadas, subtropicales y tropicales. Se alimenta de granos de maíz partido, cacao, yuca seca, ñame, leguminosas y nuez moscada (Tróchez 1987). Tiene preferencia por granos húmedos y mohosos, en sitios con alta humedad (> $70 \%$ ). El adulto es de color castaño rojizo, de 1,5 a 1,8 mm de longitud, similar en apariencia a los adultos de $C$. quadricollis pero de menor tamaño, con el cuerpo más ovalado y pronoto ligeramente transverso, embombado y ribeteado con los bordes laterales finamente dentados. Ambas especies son consideras plagas secundarias porque se alimentan de granos almacenados.

Las especies de Silvanidae se caracterizan por ser depredadores de ácaros y pequeños insectos, así como por ubicarse frecuentemente en lugares escondidos. Esta es probablemente la razón por la que se encuentran estas especies dentro de los frutos de café, aprovechando el orificio realizado durante la entrada de la broca, con el fin de buscar refugio y estados inmaduros de la broca como fuente de alimentación (Vera et al. 2007).

En unas pruebas de palatabilidad realizadas en Cenicafé con diferentes dietas, se estudió el comportamiento de alimentación y biología de C. quadricollis y A. advena y se concluyó que estas especies no consumen ni se reproducen en almendra molida de café (Cenicafé 2013). Esto confirma que su presencia en las galerías de la broca del café está relacionada con eventos de depredación de estados inmaduros de broca y no representan riesgos como potenciales plaga del café.

Teniendo en cuenta estos antecedentes, se llevó a cabo esta investigación con el objetivo de evaluar la capacidad de C. quadricollis y A. advena de disminuir las poblaciones de la broca del café al interior de granos de café infestados. Como hipótesis de trabajo se estableció que estas dos especies tenían la capacidad de entrar a los frutos de café, depredar los estados de la broca en su interior y disminuir las poblaciones de la plaga, siendo mayor la disminución en la medida que se aumentara la proporción de depredadores por grano infestado.

\section{Materiales y métodos}

Para el establecimiento de las colonias de cría de $C$. quadricollis y A. advena, se recolectaron granos de café infestados por broca en las Estaciones Experimentales de Cenicafé: Naranjal, La Catalina y Paraguaicito, así como en tres fincas particulares cuya ubicación y altitud se resumen en la Tabla 1. En cada localidad se recolectaron 500 frutos secos de los árboles y 500 del suelo, para un total de 6.000. Al momento de la recolección de los frutos, los niveles de infestación por broca se encontraban entre 4 y $10 \%$.

Para la obtención de los adultos de A. advena y C. quadricollis, los frutos de café secos brocados de cada lote se depositaron en recipientes plásticos de $22 \mathrm{~cm} \mathrm{x} 11 \mathrm{~cm} \times 7$ $\mathrm{cm}$, cubiertos con tapa y dos orificios circulares de ventila-

Tabla 1. Sitios muestreados para la obtención de enemigos naturales de la broca del café.

\begin{tabular}{llllll}
\hline $\begin{array}{l}\text { Finca/ } \\
\text { estación }\end{array}$ & Departamento & Municipio & $\begin{array}{c}\text { Altitud } \\
\text { (msnm) }\end{array}$ & $\begin{array}{c}\text { Latitud } \\
\text { norte }\end{array}$ & $\begin{array}{c}\text { Longitud } \\
\text { oeste }\end{array}$ \\
\hline Paraguaicito & Quindío & Buenavista & $04^{\circ} 24^{\prime}$ & $75^{\circ} 44^{\prime}$ & $04^{\circ} 24^{\prime}$ \\
La Bella & Quindío & Calarcá & $04^{\circ} 30^{\prime}$ & $75^{\circ} 40^{\prime}$ & $04^{\circ} 30^{\prime}$ \\
La Catalina & Risaralda & Pereira & $04^{\circ} 45^{\prime}$ & $75^{\circ} 44^{\prime}$ & $04^{\circ} 45^{\prime}$ \\
Naranjal & Caldas & Chinchiná & $04^{\circ} 58^{\prime}$ & $75^{\circ} 39^{\prime}$ & $04^{\circ} 58^{\prime}$ \\
Las Carolas & Caldas & Chinchiná & $04^{\circ} 59^{\prime}$ & $75^{\circ} 37^{\prime}$ & $04^{\circ} 59^{\prime}$ \\
La Paloma & Caldas & Palestina & $05^{\circ} 01^{\prime}$ & $75^{\circ} 38^{\prime}$ & $05^{\circ} 01^{\prime}$ \\
\hline
\end{tabular}


ción de $3 \mathrm{~cm}$ de diámetro forrados con muselina blanca. Estos fueron mantenidos hasta la emergencia de los depredadores en un cuarto climatizado a $24^{\circ} \mathrm{C}$ y $76 \%$ HR. Para la recolección y separación de los adultos, se realizó un tamizaje y cernido del ripio de café con malla metálica de $0,5 \mathrm{~mm} \times 0,5$ $\mathrm{mm}$ de diámetro cada 15 días. Una vez obtenidos los adultos se establecieron las colonias de cría de las dos especies bajo condiciones de laboratorio $\left(24^{\circ} \mathrm{C}\right.$ y $\left.70 \% \mathrm{HR}\right)$ en la planta piloto de Cenicafé, en Manizales, Caldas. Se usaron recipientes de cría plásticos y de vidrio dentro de los cuales se pusieron individuos adultos de cada depredador alimentados con granos de maíz trillado. Con el fin de garantizar el suministro continuo de alimento y evitar contaminación de las crías, se cambió el alimento cada 30 días.

Para evaluar la capacidad de los depredadores la disminución de las poblaciones de la broca al interior de los granos de café infestados por la broca, se realizaron cuatro bioensayos independientes, liberando adultos y larvas L3 de las dos especies de insectos. Para cada bioensayo se conformaron unidades experimentales con 10 granos de café pergamino húmedo al $47 \%$, infestados 18 días antes con adultos de broca. Se seleccionaron aquellos que contenían tres orificios de entrada, con un promedio 8 a 10 estados biológicos de broca. Los granos se depositaron en frascos de vidrio, cubierto con muselina blanca. Posteriormente se liberaron los depredadores en tres proporciones (tratamientos): $\mathrm{T}_{1}: 1, \mathrm{~T}_{2}: 3 \mathrm{y} \mathrm{T}_{3}: 5$ depredadores por grano infestado. Se estableció un tratamiento control $\mathrm{T}_{0}$ donde no se liberaron depredadores. Cada bioensayo fue establecido bajo un diseño completamente aleatorio con 10 repeticiones. Se registró el número de estados vivos de la broca en cada grano infestado de cada unidad experimental. Como variable de respuesta se utilizó el total de estados de broca vivos (TE) y como variables complementarias el total de estados vivos de broca sin adultos (TESA) y la depreda- ción de cada estado biológico de la broca. Esto con el fin de descartar los estados adultos de la broca, los cuales, en las pruebas preliminares de laboratorio, no fueron depredados.

Los datos se analizaron por medio de la estimación del promedio y del coeficiente de variación por tratamiento, con un análisis de varianza al $5 \%$. De igual forma, se compararon los tratamientos frente al testigo absoluto $\left(\mathrm{T}_{0}\right)$ con la prueba de Dunnett. Aquellos que difirieron del testigo se les realizó un nuevo análisis de varianza y los que mostraron efecto de tratamientos se sometieron a una evaluación de tendencia lineal y cuadrática para determinar la mejor proporción depredador a grano. Todos los análisis se realizaron con el programa estadístico SAS (SAS Institute 2007). La hipótesis de trabajo se corroboraría siempre y cuando se encontraran diferencias estadísticas entre tratamientos y si la disminución de la broca fuese mayor cuando se liberaron más de 1 depredador por grano infestado por broca.

\section{Resultados y discusión}

Se encontraron diferencias estadísticas en el total de estados de broca vivos cuando se liberaron las dos especies de los depredadores en sus dos estados de vida, adultos y larvas. La liberación de adultos de $A$. advena permitió registrar diferencias significativas entre los tratamientos $(\mathrm{F}=39,18$; g.1. $=3 ; \mathrm{P}<0,001)$ para la variable TE (Tabla 2). La prueba de Dunnett mostró que todos los tratamientos con adultos fueron estadísticamente diferentes al testigo (Tabla 2). La mayor tasa de depredación de estados de broca fue del 63,2\%, obtenida en el tratamiento $\mathrm{T}_{2}$ con liberación de tres depredadores adultos por grano (Tabla 3 ). La evaluación de tendencias entre los tratamientos con uno, tres y cinco depredadores no fue significativa para el tipo lineal $(\mathrm{F}=3,63 ; \mathrm{g} .1,=1 ; \mathrm{P}=0,0674)$ ni cuadrático $(\mathrm{F}=1,38 ; \mathrm{g} . \mathrm{l}=1 ; \mathrm{P}=0,2497)$, es decir, ninguno

Tabla 2. Número de estados vivos de broca, considerando los adultos (TE) y excluyéndolos (TESA), posterior a la liberación de adultos y larvas de Ahasverus advena y Cathartus quadricollis en las proporciones de uno, tres y cinco depredadores por grano infestado. "Control" se refiere al testigo sin depredadores y E1 y E2 al Error estándar de la variable TE y TESA, respectivamente, $(\mathrm{n}=10)$.

\begin{tabular}{|c|c|c|c|c|c|}
\hline Estado de especie depredadora & $\begin{array}{c}\text { Número de } \\
\text { depredadores / grano }\end{array}$ & TE & E1 & TESA & $\mathbf{E 2}$ \\
\hline \multirow{4}{*}{ Ahasverus advena - Adultos } & Control & 346,8 & 25,05 & 161,8 & 14,43 \\
\hline & 1 & $163,4^{*}$ & 15,94 & $56,3^{*}$ & 7,36 \\
\hline & 3 & $127,7^{*}$ & 10,33 & $20,0 *$ & 3,57 \\
\hline & 5 & $128,9^{*}$ & 11,44 & $20,4 *$ & 5,29 \\
\hline \multirow{4}{*}{ Ahasverus advena - Larvas } & Control & 244,9 & 18,56 & 206,6 & 14,13 \\
\hline & 1 & 184,1 & 24,40 & $150,2^{*}$ & 19,51 \\
\hline & 3 & $141,3 *$ & 13,84 & $112,1^{*}$ & 9,93 \\
\hline & 5 & $147,5^{*}$ & 13,98 & $120,8^{*}$ & 11,09 \\
\hline \multirow{4}{*}{ Cathartus quadricollis - Adultos } & Control & 192,8 & 17,82 & 149,1 & 15,53 \\
\hline & 1 & 170,6 & 20,18 & 132,1 & 17,99 \\
\hline & 3 & $103,7 *$ & 10,26 & $71,7^{*}$ & 7,61 \\
\hline & 5 & 147,2 & 10,87 & 105,4 & 9,35 \\
\hline \multirow{4}{*}{ Cathartus quadricollis - Larvas } & Control & 242,6 & 12,28 & 191,7 & 8,90 \\
\hline & 1 & $165,9^{*}$ & 21,96 & $127,3^{*}$ & 18,79 \\
\hline & 3 & $104,4^{*}$ & 10,37 & $77,0^{*}$ & 8,89 \\
\hline & 5 & $75,3^{*}$ & 9,71 & $46,8^{*}$ & 9,33 \\
\hline
\end{tabular}

* Promedio significativamente diferente respecto a su testigo, de acuerdo a prueba Dunnett al $5 \%$. 

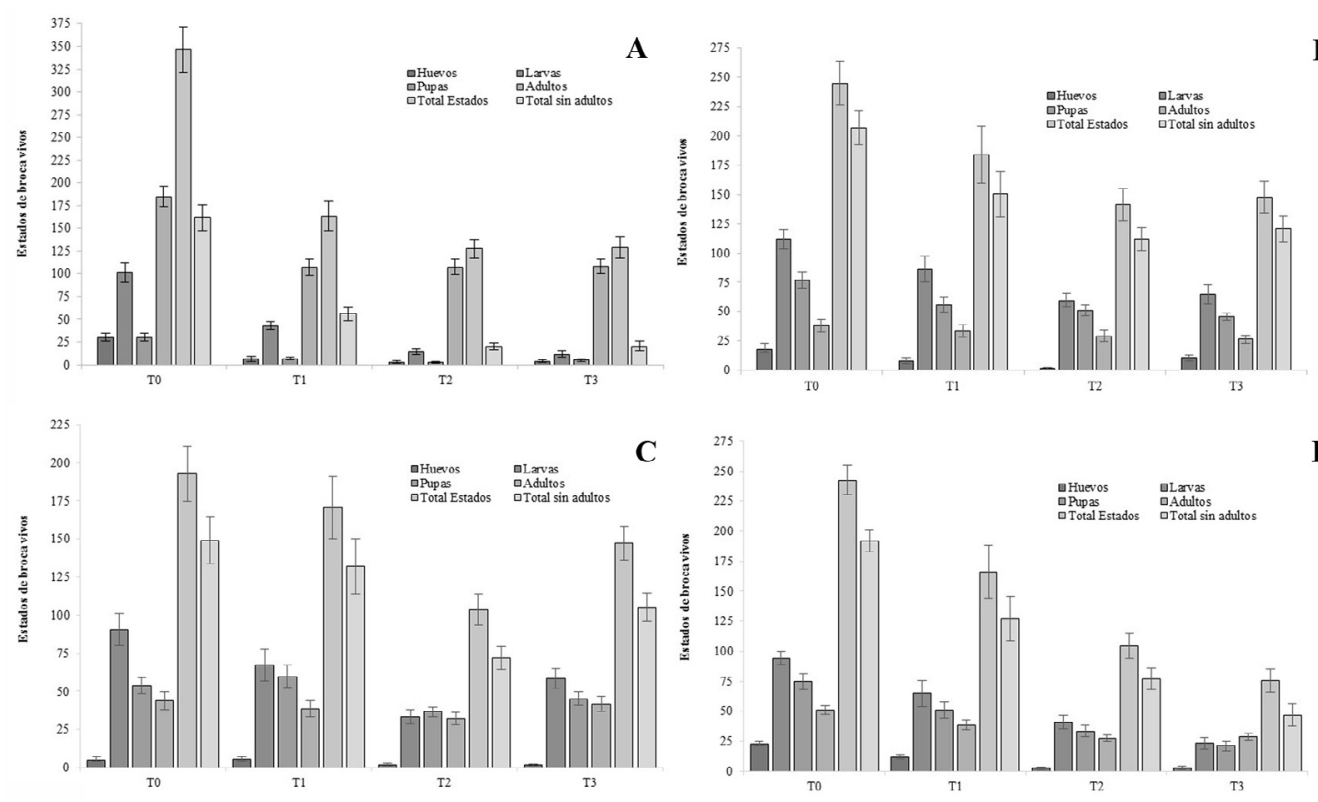

Figura 1. Promedios y error estándar del número de estados biológicos de broca vivos después de 10 días de liberados A. Adultos de A. advena. B. Larvas de A. advena. C. Adultos de C. quadricollis. D. Larvas de C. quadricollis. $\mathrm{n}=10$

de los dos modelos logró explicar el comportamiento de la variable TE. Sin embargo, para la variable complementaria TESA se presentó tanto una tendencia lineal $(\mathrm{F}=20,37$; g.l. $=$ $1 ; \mathrm{P}<0,001)$ como cuadrática $(\mathrm{F}=7,10 ;$ g.l. $=1 ; \mathrm{P}=0,0129)$. Los resultados permiten sugerir que la proporción de tres y cinco adultos del depredador $A$. advena permite una mayor disminución de las poblaciones de broca del café al interior de los frutos infestados.

La evaluación de larvas de $A$. advena igualmente mostró diferencias significativas entre los tratamientos $(\mathrm{F}=6,81$; g.l. $=3 ; \mathrm{P}<0,001)$ para la variable TE (Tabla 2). La prueba de Dunnett mostró que los tratamientos $\mathrm{T}_{2} \mathrm{y} \mathrm{T}_{3}$ fueron estadísticamente diferentes al testigo (Tabla 2). La mayor disminución de los estados de la broca fue del $42,3 \%$ obtenida en el $\mathrm{T}_{2}$ (Tabla 3). Sin embargo, al no encontrarse diferencia entre $\mathrm{T}_{2} \mathrm{y} \mathrm{T}_{3}$ para la variable TE $(\mathrm{F}=0,10$ g.l. $=1 ; \mathrm{P}=0,7563)$ y tampoco entre los tratamientos $\mathrm{T}_{1}, \mathrm{~T}_{2}$ y $\mathrm{T}_{3}$ para la variable TESA $(\mathrm{F}=1,99 ;$ g.l. $=2 ; \mathrm{P}=0,1568)$, no fue necesario realizar el análisis de tendencia y es posible concluir que la liberación de tres larvas por grano infestado sería suficiente para disminuir las poblaciones de la broca al interior de los frutos infestados.

Con respecto a $C$. quadricollis, la capacidad de los adultos de disminuir las poblaciones de broca permitió establecer diferencias significativas entre tratamientos $(\mathrm{F}=6,13$; g.l. $=$ 3; $\mathrm{P}=0,0018$ ) para la variable TE (Tabla 2). La prueba de Dunnett mostró que el tratamiento $\mathrm{T}_{2}$ fue estadísticamente diferente al testigo T0 (Tabla 2), obteniéndose una disminución del 46,2 \% de los estados de la broca en los frutos (Tabla 3 ). La evaluación de tendencias entre los tratamientos $\mathrm{T}_{1}, \mathrm{~T}_{2} \mathrm{y} \mathrm{T}_{3}$ para la variable TESA mostró un comportamiento cuadrático $(\mathrm{F}=9,44 ;$ g.l. $=1 ; \mathrm{P}=0,0048)$, lo que indica que el trata-

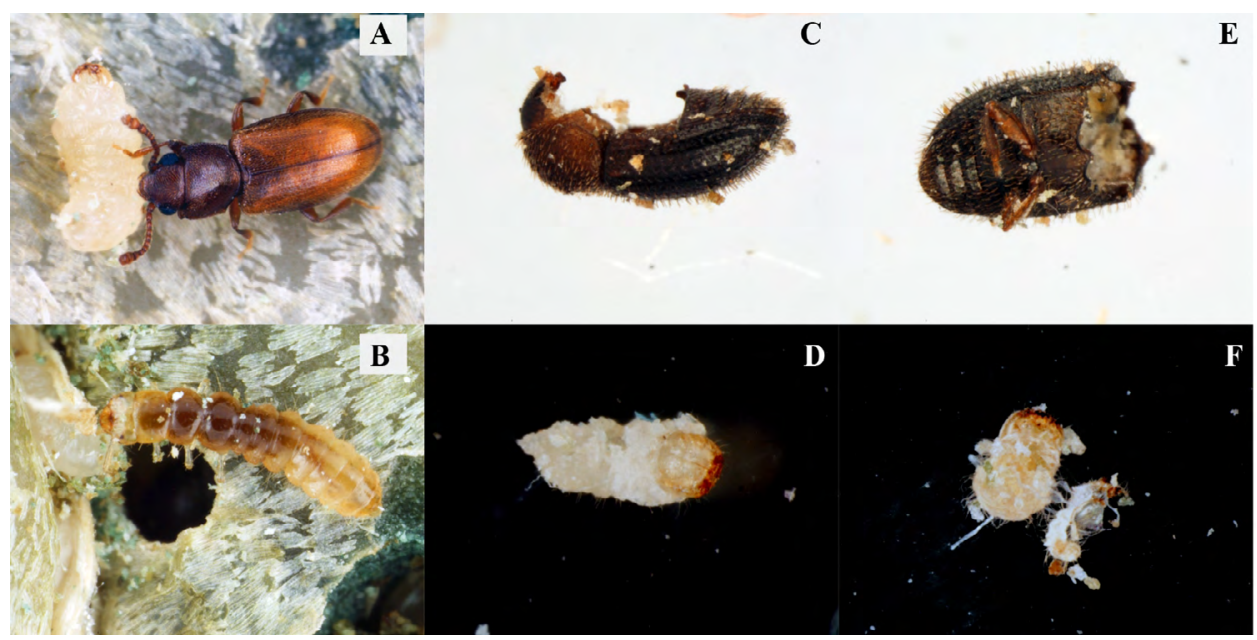

Figura 2. Adulto (A) y larva (B) de Ahasverus advena depredando larvas de broca. C, E. Adultos. D, F. larvas de broca parcialmente depredadas por adultos de Ahasverus advena. 
Tabla 3. Porcentajes de diminución de estados de broca con respecto a un testigo sin depredadores, posterior a la liberación de adultos y larvas de los depredadores Ahasverus advena y Cathartus quadricollis después de 10 días de exposición $(\mathrm{n}=10)$.

\begin{tabular}{lccc}
\hline Estado de especie depredadora & $\begin{array}{c}\text { Número de depredadores } \\
\text { / grano }\end{array}$ & \% TE & \% TESA \\
\hline \multirow{3}{*}{ Ahasverus advena - Adultos } & 1 & 52,9 & 65,2 \\
& 3 & 63,2 & 87,6 \\
& 5 & 62,8 & 87,4 \\
\hline \multirow{2}{*}{ Ahasverus advena - Larvas } & 1 & 24,8 & 27,3 \\
& 3 & 42,3 & 45,7 \\
Cathartus quadricollis - Adultos & 5 & 39,8 & 41,5 \\
\hline \multirow{2}{*}{ Cathartus quadricollis - Larvas } & 1 & 11,5 & 11,4 \\
& 3 & 46,2 & 51,9 \\
& 5 & 23,7 & 29,3 \\
\hline
\end{tabular}

miento que ofreció mejores resultados fue la liberación de tres adultos del depredador por grano.

La evaluación de larvas de $C$. quadricollis igualmente mostró diferencias significativas entre tratamientos $(\mathrm{F}=$ 26,28; g.1. $=3 ; \mathrm{P}<0,001)$ (Tabla 2). La mayor disminución de estados de broca fue del $69,0 \%$ en el tratamiento $\mathrm{T}_{3}$ con 5 depredadores por grano (Tabla 3). La evaluación de tendencias entre los tratamientos $\mathrm{T}_{1}, \mathrm{~T}_{2}$ y $\mathrm{T}_{3}$ mostró un comportamiento lineal de la variable $\mathrm{TE}(\mathrm{F}=18,00 ;$ g.l. $=1 ; \mathrm{P}=$ $0,0002)$, lo cual indica que cinco larvas del depredador por grano fue el más efectivo reduciendo las poblaciones de la broca dentro de los granos infestados.

La liberación de las dos especies de depredadores, tanto en adultos como en larvas, mostró una preferencia hacia los estados inmaduros de la broca, principalmente las larvas y los huevos (Figs. 1 y 2); sin embargo, se observaron adultos depredados (Figs. 2C y 2E). Se considera que el consumo de las pupas de la broca se pudo haber reflejado en la diferencia entre el número de adultos de broca vivos en cada bioensayo con respecto a su testigo, ya que el número de pupas consumidas equivaldría al número de individuos que, de no haber sido depredados en su estado pupal, hubieran formado adultos una vez pasados los 10 días de exposición de los granos.

Los resultados obtenidos en este estudio permitieron corroborar parcialmente la hipótesis de trabajo ya que se encontraron diferencias estadísticas entre tratamientos cuando se liberaron los adultos y las larvas de A. advena y C. quadricollis. A pesar de que sólo las larvas de C. quadricollis presentaron una tendencia lineal en el total de estados de broca al interior de los frutos infestados, es decir, a mayor proporción de depredadores liberados mayor disminución en las poblaciones de broca, se pudo establecer que la liberación de tres depredadores por grano infestado, tanto de adultos como larvas de $A$. advena, y de cinco adultos de C. quadricollis, permiten disminuir todos los estadíos de las poblaciones de la broca del café en los frutos infestados entre un $42 \%$ y un $69 \%$.

Estos son los primeros resultados de laboratorio con el uso de depredadores nativos para el control biológico de la broca del café en Colombia, los cuales aún no permiten recomendar estas especies dentro de una estrategia de control biológico. Sin embargo, estos resultados abren una nueva posibilidad en el manejo integrado de esta plaga limitante de la producción y calidad del café. Es por esto necesario identificar los riesgos asociados a las liberaciones masivas de depredadores en una estrategia de control biológico por aumentación, dado que se podrían desplazar especies no blanco, depredar otros insectos benéficos (Chang y Kareiva 2004), producir molestias a comunidades humanas por aumentos exagerados de sus poblaciones (Kovach 2004) o alimentarse de especies botánicas de interés económico (Tróchez 1987). La literatura reciente, a pesar de considerar estos riesgos (Simberloff y Stiling 1996), también registra experiencias de impactos positivos con el uso de coleópteros nativos omnívoros como controladores biológicos (Kromp 1999; Symondson et al. 2002). Por su parte, Kanat y Özbolat (2006) criaron y liberaron a Calosoma sycophanta (Linnaeus, 1758) (Coleoptera: Carabidae) como agente de control biológico de Thaumetopoea pityocampa (Schiff, 1986) (Lepidoptera: Thaumetopoeidae) en Kahramanmarafl, Turquía, sin reportar efectos negativos.

Consecuentemente, se recomienda evaluar en condiciones de campo, en bioensayos de exclusión, la capacidad de estos depredadores de disminuir la broca y los niveles de infestación. La variable de interés deberá ser la infestación ocasionada por la broca en los frutos de café de las cosechas principales, posterior a la liberación de los depredadores una vez finalice la cosecha inmediatamente anterior. Se recomienda evaluar previamente el efecto de los adultos y las larvas sobre los frutos infestados por broca en el suelo y en el árbol.

\section{Agradecimientos}

Los autores manifiestan sus agradecimientos al laboratorio Biocafé por suministrar el pie de cría de los depredadores usados en este estudio, a Esther Cecilia Montoya por su acompañamiento estadístico y al auxiliar de investigación Juan Paulo Pimentel. 


\section{Literatura citada}

BENAVIDES M., P.; GIL P., Z. N.; CONSTANTINO, L. M.; VILLEGAS, C.; GIRALDO, M. 2013. Manejo integrado de la broca del café Hypothenemus hampei (Ferrari) (Coleoptera: Curculionidae) pp. 216-225. En: Manual del cafetero Colombiano. Tomo 2. Investigación y tecnología para la sostenibilidad de la caficultura colombiana. Chinchiná, Cenicafé, Editorial Legis, Bogotá, Colombia. 354 p.

BUSTILLO, A. E. 2007. El manejo de cafetales y su relación con el control de la broca del café en Colombia. Boletín técnico No. 24. $2^{\mathrm{a}}$ edición. Chinchiná, Cenicafé. $40 \mathrm{p}$.

BUSTILlO, A. E.; CARDENAS, R.; VILlaLBA, D. A.; BENAVIDES M, P.; OROZCO, J.; POSADA, F. J. 1998. Manejo integrado de la broca del café, Hypothenemus hampei (Ferrari) en Colombia. Chinchiná, Cenicafé, Editorial Feriva, Cali, Colombia. 134 p.

BUSTILLO P., A. E.; CÁRDENAS M., R.; POSADA F., F. J. 2002. Natural enemies and competitors of Hypothenemus hampei (Ferrari) (Coleoptera: Scolytidae) in Colombia. Neotropical Entomology 31 (4): 635-639.

CENICAFÉ, 2013. Cría e identificación de enemigos naturales de la broca del café. pp. 49-52. Informe anual Cenicafé.

CHANG, G. C.; KAREIVA, P. 2004. The case for indigenous generalists in biological control. pp. 103-115. In: Hawkins, B. A.; Cornell, H. V. (Eds.). Theoretical approaches to biological control. Cambridge University Press. New York. EE. UU. 426.

FOLLET, P. A.; KAWABATA, A.; NELSON, R.; ASMUS, G.; BURT, J.; GOSCHKE, K.; EWING, C.; GAERTNER, J.; BRILL, E.; SCOTT, G. 2016. Predation by flat bark beetles (Coleoptera: Silvanidae and Laemophloeidae) on coffee berry borer (Coleoptera: Curculionidae) in Hawaii coffee. Biological Control 101: 152-158.

JARAMILLO, J. 2008. Biology, ecology and biological control of the coffee berry borer, Hypothenemus hampei (Ferrari) (Coleoptera: Curculionidae: Scolytinae). Ph. D. thesis, Faculty of Natural Sciences, Gottfried Wilhelm Leibniz Universität Hannover, Alemania. 237 p.

KANAT, M.; ÖZBOLAT, M. 2006. Mass production and release of Calosoma sycophanta L. (Coleoptera: Carabidae) used against the pine processionary moth, Thaumetopoea pityocampa
(Schiff.) (Lepidoptera: Thaumetopoeidae), in biological control. Turkish Journal of Zoology 30: 181-185.

KOVACH, J. 2004. Impact of the multicolored Asian lady beetle as a pest of fruit and people. American Entomologist 50 (3): 159-161

KROMP, B. 1999. Carabid beetles in sustainable agriculture: a review on pest control efficacy, cultivation impacts and enhancement. Agriculture, Ecosystems and Environment 74: 187-228.

PÉREZ-LACHAUD, G. 1998. A new bethylid attacking the coffee berry borer in Chiapas (Mexico) and some notes on its biology. Southwestern Entomologist 23: 287-288.

SAS INSTITUTE INC. 2007. JMP statistical discovery software. Version 7. SAS Institute Inc., Cary, North Carolina.

SIMBERLOFF, D.; STILING, P. 1996. How risky is biological control? Ecology 77 (7): 1965-1974.

SYMONDSON, W. O. C.; SUNDERLAND, K. D.; GREENSTONE, M. H. 2002. Can generalist predators be effective biocontrol agents?. Annual Review of Entomology 47: 561-594.

TRÓCHEZ, A. 1987. Manual de reconocimiento de insectos asociados con productos almacenados. IICA. Bogotá. 123 p.

VEGA, F. E.; INFANTE, F.; CASTILLO, A.; JARAMILLO, J. 2009. The coffee berry borer, Hypothenemus hampei (Ferrari) (Coleoptera: Curculionidae): a short review, with recent findings and future research directions. Terrestrial Arthropod Reviews 2: 129-147.

VERA, L. Y.; GIL, Z. N.; BENAVIDES M., P. 2007. Identificación de enemigos naturales de Hypothenemus hampei en la zona cafetera central colombiana. Cenicafé 58 (3): 185-195.

Recibido: 2-jun-2017 • Aceptado: 25-may-2018

Citación sugerida:

LAITON J, L. A.; CONSTANTINO, L. M.; BENAVIDES, P. 2018. Capacidad depredadora de Cathartus quadricollis y Ahasverus advena (Coleoptera: Silvanidae) sobre Hypothenemus hampei (Coleoptera: Curuculionidae) en laboratorio. Revista Colombiana de Entomología 44 (2): 200-205. Julio-Diciembre 2018. 